\title{
Yod
}

Revue des études hébraïques et juives

$16 \mid 2011$

Le yiddish dans la sphère francophone

\section{Le yiddish : un passé proche et un souvenir éternel dans l'univers d'Aharon Appelfeld}

Yiddish: a recent past and an eternal memory in the world of Aharon Appelfeld

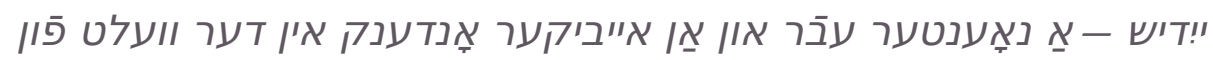

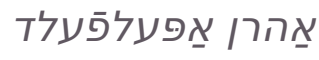

\section{Masha Itzhaki}

\section{OpenEdition}

Journals

Édition électronique

URL : https://journals.openedition.org/yod/655

DOI : $10.4000 /$ yod.655

ISSN : 2261-0200

Éditeur

INALCO

\section{Édition imprimée}

Date de publication : 1 juin 2011

Pagination : $51-59$

ISBN : 978-2-85831-191-0

ISSN : 0338-9316

\section{Référence électronique}

Masha Itzhaki, « Le yiddish : un passé proche et un souvenir éternel dans l'univers d'Aharon Appelfeld », Yod [En ligne], 16 | 2011, mis en ligne le 06 décembre 2011, consulté le 08 juillet 2021. URL : http://journals.openedition.org/yod/655 ; DOI : https://doi.org/10.4000/yod.655

Ce document a été généré automatiquement le 8 juillet 2021.

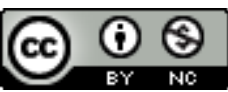

Yod est mis à disposition selon les termes de la Licence Creative Commons Attribution - Pas d'Utilisation Commerciale 4.0 International. 


\title{
Le yiddish : un passé proche et un souvenir éternel dans l'univers d'Aharon Appelfeld
}

\author{
Yiddish: a recent past and an eternal memory in the world of Aharon Appelfeld

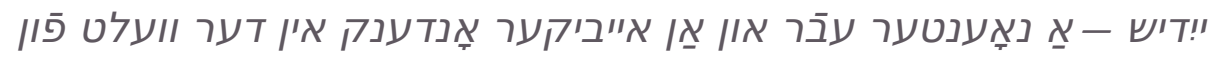

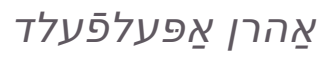

\section{Masha Itzhaki}

1 Selon le premier chapitre d'Histoire d'une vie ${ }^{1}$, l'ouvrage semi-autobiographique d'Aharon Appelfeld, un seul mot prononcé par sa mère constitue le premier souvenir clair d'un enfant de quatre ans, Erdbeeren, «fraises» en allemand, en hébreu, tut sade. Cet enfant est élevé dans un environnement multilingue, caractéristique du milieu juif de l'époque: il parle allemand avec sa mère, la langue de ses grands-parents est le yiddish, tandis que la population environnante s'exprime en ukrainien. De plus, la langue de Dieu, celle de la prière, l'hébreu donc, qu'il entend à la synagogue où il va avec son grand-père lorsqu'il est en vacances chez ses grands-parents, est un grand mystère aux yeux du petit : "Deux enfants de mon âge se tiennent debout comme des adultes et prient. Ils savent déjà comment parler à Dieu, et moi, je suis muet ${ }^{2}$.»

2 «J'ai appris le yiddish pour qu'il me soit plus facile d'adopter l'hébreu », dit Appelfeld quand il raconte le parcours difficile de l'apprentissage de l'hébreu. Lors d'une rencontre à Jérusalem en 2009, il ajoute : « Un auteur juif doit connaître le yiddish, cela est indispensable. » Cette langue, il l'a entendue assez souvent, enfant, chez ses grandsparents à la campagne même si ses parents ne la parlaient jamais. Cette rupture entre l'allemand en ville et le yiddish à la campagne, le petit Erwin la devine devant le malaise de sa mère qui ne parle plus que l'allemand, prétendant avoir oublié ${ }^{3}$ la langue de ses parents. Il s'agit là d'une manifestation sociolinguistique de la crise culturelle du judaïsme européen à l'époque moderne. Les Juifs assimilés, vivant dans les grands centres urbains, les gens du monde, aisés et instruits, méprisaient la langue du shtetl, 
celle de la bourgade juive traditionnelle d'Europe de l'Est et l'abandonnaient volontiers. Pourtant, cette rupture perd tout son sens avec la guerre :

On n'oubliera jamais les expressions de stupéfaction des Juifs allemands et autrichiens, pour la plupart assimilés depuis des générations, qui furent exilés dans les ghettos de l'Est. Ils rencontrèrent là, avec un immense étonnement, les Juifs yiddish qu'ils s'étaient efforcés d'ignorer depuis tant d'années. La main de Satan fit ce que, seule, elle pouvait faire: les ramener à l'endroit même qu'ils souhaitaient fuir ${ }^{4}$.

Appelfeld, l'adolescent d'après-guerre, n'a pas adopté l'option du refoulement et de l'oubli afin de devenir un "vrai " sabra. Bien au contraire, il a choisi sciemment de rétablir les liens avec son passé et sa famille disparue. À l'université, à l'encontre du «bon ton» de l'époque, il décide d'étudier le yiddish, cette langue "qui bruissait encore des souvenirs de la maison de grand-père ", qui symbolisait encore la diaspora et la faiblesse et qui était, comme il le dit, orpheline tout comme lui. Cette décision lui est entièrement bénéfique. Son professeur, Dov Sadan ${ }^{5}$, le plus grand connaisseur de l'histoire de la littérature juive en yiddish et en hébreu, est devenu pour le jeune Appelfeld une sorte de guide spirituel qui lui a fait connaître l'ensemble de l'univers culturel juif : le hassidisme, la Haskala, le Bund et le sionisme. « Pour la première fois de ma vie, j'ai eu une orientation juive. Chez lui, un cours n'était en fait qu'un long dialogue ", raconte-t-il. Le choix du yiddish n'est pas dû au hasard. Il confirme les liens toujours existants avec ses grands-parents et avec le passé, en constituant un contrepoids au mythe du sabra.

Vingt-cinq ans plus tard, Appelfeld, déjà célèbre, consacre à Dov Sadan un essai intitulé Le maître $\left(\mathrm{Ha}-\right.$ moreh $\left.^{6}\right)$. Sur les marches du bâtiment Terra Sancta à Jérusalem, là où les cours avaient lieu dans les années cinquante, il lui a montré le chemin du retour vers sa propre âme perplexe, vers son peuple. Appelfeld lui en sera reconnaissant toute sa vie.

5 Le jeune homme étudie également la poésie hébraïque avec la poétesse Léa Goldberg ${ }^{7}$ et le professeur Arieh Ludwig Strauss ${ }^{8}$, tous deux originaires du monde germanique et conscients du problème d'une double patrie. Attiré par la kabbale et le hassidisme, il a suivi les cours de Scholem, un «magicien» selon ses propres termes, et de Martin Buber qu'il qualifie de mélange «entre un rebe hassidique et un professeur des universités à l'allemande. » L'université de l'époque, composée d'enseignants de culture allemande et d'étudiants déjà adultes, anciens combattants d'une part et rescapés de la Shoah de l'autre, a su accueillir ce jeune homme si seul. L'adulte les remercie ainsi :

Les années à la maison et celles de la guerre ont formé mes instincts et mes sensations. Les années à l'université ont formé mon sens critique et mon style. J'ai eu de la chance: mes professeurs sont devenus mes guides, j'ai continué à les voir même après les études. Ils connaissaient mes tourments d'écrivain et ils m'ont dit la vérité. Leur critique n'a pas toujours été facile à accepter. Quand ma nouvelle "Comme la prunelle de l'œil »" est parue, Scholem m'a serré les mains entre les siennes et m'a dit : "Appelfeld, tu es un écrivain ${ }^{10}$."

6 Avec son maître Dov Sadan, il s'initie par le biais du yiddish à la vaste culture juive et fait connaissance avec le hassidisme. À ce propos, il évoque souvent l'écrivain yiddish Leib Rochman (1918-1978) chez qui il a trouvé une seconde maison. Rochman lui a fait connaître le yiddish dans toute sa pureté ainsi qu'un regard particulier sur l'univers hassidique. Il était pour lui à la fois un frère aîné et un père spirituel et sa maison à Jérusalem, lieu de rencontre des hommes de lettres et des universitaires, se substitue au cadre familial perdu. Quand en 1978, à la veille de son décès, le dernier recueil des nouvelles de Rochman voit le jour, Appelfeld y ajoute un magnifique texte de conclusion. 
7 Le choix du yiddish comme matière principale dans le parcours universitaire du jeune homme signale, semble-t-il, la première étape dans un long processus identitaire particulièrement douloureux : la déconstruction du mythe $d u$ "Juif nouveau » pour établir une identité plus complexe et plus riche de l'Israélien d'aujourd'hui. Celui-ci est le fruit d'un présent, certes, mais aussi d'un passé proche et surtout, consciemment ou non, d'une très longue histoire.

Nous pensions qu'en venant ici, en Israël, nous serions normaux, un peuple parmi les autres. Or, cette pensée était naïve, comme s'il était possible de changer un homme, une société, un mode de vie séculaire d'un coup de baguette magique. C'est impossible. C'était une naïveté d'idéalistes : devenir normaux, blonds, grands... Grâce à Dieu, nous sommes restés les mêmes ${ }^{11}$.

Et Appelfeld ajoute :

La plupart des gens de ce pays sont des immigrés, eux ou leurs parents. [...] Et c'est très bien. L'immigré apporte avec lui deux univers, deux identités, deux langues, deux paysages. Il a une perspective. [...] Le Juif n'a jamais vécu dans une seule dimension. Le fait que le pays se développe, progresse, est dû aux immigrés, à leur mouvement incessant, à leur imagination et à leur énergie.

Dans ses romans, Appelfeld parle du yiddish à maintes reprises. À titre d'exemple, Helga, la narratrice de Toute une vie ${ }^{12}$, nous parle de deux langues, le yiddish et l'hébreu, qu'il est à ses yeux obligatoire d'acquérir afin d'intérioriser la foi juive, celle de sa mère. Dans le centre médical pour les survivants de la Shoah où elle travaille à la fin de la guerre, Helga apprend l'hébreu et le yiddish, les deux langues sacrées selon ses propos. Elle apprend aussi à chanter des chants populaires yiddish tout comme des prières ou des chansons sionistes en hébreu, comme si inconsciemment, par un fil mystérieux, elle avait entendu les derniers propos de sa mère au camp tels qu'ils sont rapportés par une de rescapées :

Gisela était notre lumière. Elle nous a enseigné des mots en hébreu et en yiddish. Après une journée difficile ou une punition collective, elle nous a récité avec beaucoup d'émotion la prière du Shema. Sais-tu de quoi il s'agit? Sais-tu quelle puissance il faut pour frayer un chemin aux cours des gens désespérés ${ }^{13}$ ?

Ce sont donc des chansons en yiddish et en hébreu qui accompagnent Gisela lors de son enterrement dans les ruines de l'ancien cimetière juif à proximité de la ville.

Mais l'œuvre principale concernant cette langue est sans aucun doute le roman Une nuit et encore une autre ${ }^{14}$ paru en 2001, où le yiddish est non seulement le thème principal, mais aussi le héros du récit. L'intrigue se déroule dans une pension de famille dans la Jérusalem des années cinquante. Tous les pensionnaires sont des survivants de la Shoah qui mènent un combat incessant pour faire revivre leur langue maternelle, le yiddish, et conserver ses traces écrites et orales à tout prix afin de garder à jamais en mémoire leur passé en diaspora. Parmi eux le vieil Ezéchiel, personnage qui évoque le prophète biblique, Kirtzel, le peintre qui dessine des mots en yiddish, Zeidel le poète qui colle ses poèmes sur les murs de l'établissement, Nachman Gold, le bibliothécaire qui sauve des livres, etc. Face à eux se trouve Mme Pracht, la propriétaire de la pension, une Juive cultivée d'origine allemande, qui habitait Jérusalem déjà avant la guerre. Autant les pensionnaires sont tous enthousiasmés par leur projet et fonctionnent comme s'il s'agissait d'une mission sacrée d'ordre religieux, autant la propriétaire remplit le rôle du gardien de l'ordre et du calme et se bat contre cette agitation. À la fin du roman, tout s'écroule: Mme Pracht est assassinée par l'un des pensionnaires et la petite pension ferme. 
11 Ce livre offre plusieurs lectures et il est l'un des plus profonds de la narration appelfeldienne ${ }^{15}$. Sur le plan sociohistorique, il peut se lire comme une description de la tension culturelle entre ceux qui sont venus de «là-bas», les rescapés, les pensionnaires donc, et les anciens du pays qui ont du mal à les accepter, représentés par Mme Pracht. Sur le même plan, ces deux pôles évoquent la fracture du judaïsme européen à travers le facteur linguistique : le passé chaleureux du shtetl qui s'exprime en yiddish face à la culture européenne des Juifs assimilés d'avant-guerre dont la langue était l'allemand. Le personnage de Mme Pracht représente en fait ces immigrants venus d'Allemagne en Palestine, les Yekes, totalement fidèles à leur culture d'origine. Mais cette lecture historique et sociolinguistique, tout en étant cohérente, est fondée sur une interprétation presque allégorique qui semble un peu trop simpliste. Outre le regard nettement juif, ce roman est un questionnement profond sur les liens entre la vie et la langue d'une part et l'art de l'autre. L'univers dans lequel vivent les pensionnaires est complètement déconnecté de la réalité, ils vivent dans le passé comme beaucoup de ceux qui ont subi le même sort, à la différence que ces personnages ont une démarche extrême et créent une équation irrationnelle entre la renaissance du yiddish et la résurrection des morts, comme si la langue en soi avait un pouvoir sur le déroulement de la vie. Leur raisonnement désespéré est fondé sur le modèle théologique de la résurrection : il faut à tout prix faire revivre le yiddish par l'art, car, comme le dit Zeidel le poète, le sauvetage de cette langue sacrée est le seul moyen de faire revivre les morts, le seul chemin vers le salut. L'art mène donc vers la langue qui rend possible le retour des victimes. Selon Ktzia Alon, cette structure est empruntée à la théologie chrétienne dans le sens où le sauvetage du yiddish se réfère à la résurrection du Christ. Cette idée est particulièrement intéressante, les mythes chrétiens étant ancrés dans la narration appelfeldienne. Ktzia Alon propose cependant une autre lecture, encore plus originale, qui se réfère à l'art de la propagande en général et à la propagande nazie en particulier.

Kirtzel dessine donc chaque jour un mot et le soir il présente le résultat de ses efforts dans la salle de lecture. C'est une grande peinture et le mot, semble-t-il, crie à travers la toile. Mme Pracht prétend que ce n'est que de la propagande qui n'a rien à voir avec la peinture. Seuls ceux qui ne comprennent rien à l'art sont susceptibles de l'apprécier. Mais rien n'y fait : les pensionnaires adorent les peintures de Kirtzel et ils ne sont pas les seuls, des gens viennent de Tel-Aviv et de Haïfa pour voir ces miracles ${ }^{16}$.

12 En effet, la propagande par définition n'a qu'un seul objectif : travailler sur l'opinion des masses en dépit des défauts esthétiques, son unique rôle étant de s'adresser à la foule de telle façon qu'elle reçoive le message nécessaire. Mme Pracht, bourgeoise d'origine allemande, critique sévèrement les mots dessinés qu'elle considère comme de la propagande, tandis que Kirtzel, le survivant, en fait des outils indispensables vers le salut. À la fin du livre, lorsque Mme Pracht est assassinée, Kirzel justifie l'acte en disant que «Le yiddish est une langue sacrée. Celui qui la profane doit être puni ${ }^{17}$. " Le mythe non achevé mène donc à la catastrophe, dans le passé tout comme dans le présent, et dans ce récit, l'objet mythique est le yiddish, la langue si aimée des grands-parents.

Quel message peut-on alors retirer de ces différentes lectures?

Manifestement, ce livre déborde d'amour pour cette langue et ses expressions diverses, il est plein de sympathie et de compréhension pour les pensionnaires de Mme Pracht, marginaux ou fanatiques, plongés dans un mythe impossible. Toutefois, au bout de sa longue quête, le narrateur doit se rendre à la triste évidence : le yiddish n'appartient plus à la vie quotidienne d'aujourd'hui. Tout au long du roman se dessine un conflit 
humain entre le mythe et la réalité, entre le rêve de faire revivre le passé et l'exigence de la vie au présent. C'est ainsi que Zeidel, le poète qui dirige le combat pour le yiddish, est profondément amoureux d'une jeune femme, mais celle-ci quitte Jérusalem et part définitivement en Pologne pour trouver son salut chez les catholiques qui l'ont cachée pendant la guerre; le narrateur, un homme aisé, souhaite léguer la totalité de sa fortune pour la cause sacrée, l'immortalisation du yiddish, pourtant sa contribution est refusée, ses camarades estimant que son argent sera bien plus utile à ses enfants qui sont toujours à sa charge, etc. Le message qui transparait dans ce roman pourrait donc être formulé de la façon suivante : sans aucun doute, il faut tout faire afin de conserver le yiddish comme un héritage sacré ; cette langue fait le lien avec le monde disparu, c'est évident. Mais désormais elle n'est plus la langue de la vie et ne peut absolument pas faire revivre les morts. Ce constat est la suite du parcours psychologique qui mène au sortir de la guerre à la nouvelle vie en Israël : dans la mesure même où le jeune rescapé, Erwin-Aharon Appelfeld a choisi de combattre l'oubli et retrouver le passé de son enfance afin d'être lui-même et de rester en contact permanent avec sa vérité d'adulte, il se rend compte que l'enfermement dans le passé dont le yiddish est le symbole ne mène nulle part sauf à la folie. Autrement dit, le devoir de mémoire est primordial à ses yeux, mais il ne faut pas négliger pour autant le devoir de vie. Appelfeld parle yiddish parfaitement et il le fait en public quand il en a l'occasion, mais il écrit en hébreu et seulement en hébreu. C'est un choix identitaire qui est unique en son genre, il transmet ainsi la mémoire du monde yiddish en hébreu et parvient ainsi à vaincre l'oubli à jamais.

\section{BIBLIOGRAPHIE}

ALON, Ktzia (2006), Le christianisme dans la mythopoétique d'Appelfeld, Thèse soutenue à l'Université hébraïque de Jérusalem.

APPELFELD, Aharon (1979), Essais à la première personne (מסות בגוף ראשון), Jérusalem, WZO.

APPELFELD, Aharon (1997), « Taches de mémoire » (כתמי זיכרון), Kivunim 11-12, décembre.

APPELFELD, Aharon (1999), Histoire d'une vie (סיפור חיים), Jérusalem, Keter ; Paris, Éditions de l'Olivier, 2004.

APPELFELD, Aharon (2001), Une nuit et encore une autre (לילה ועוד לילה), Jérusalem, Keter.

APPELFELD, Aharon (2006), L'héritage nu, Essais, Paris, Éditions de l'olivier (Beyond Despair, Fromm International, 1993).

APPELFELD, Aharon (2007), Toute une vie (חיים שלמים), Jérusalem, Keter.

ITZHAKI, Masha (2008), « La langue de ma mère et la langue de Dieu / le choix linguistique d'Aharon Appelfeld », Cahiers du judaïsme, juin 2008.

TAUBER, Michèle (2005), « Laila ve'od laila », REEH nº 11.

SCHNEIDER, Shmuel (2003), « Entretien avec Aharon Appelfeld » (en hébreu), Hadoar. 
SOKOLOV, Noemi (2005) « L’apprentissage de l'hébreu chez Appelfeld », Mikan 5.

\section{NOTES}

1. Aharon Appelfeld, Sippur Hayyim, Jérusalem, Keter, 1999; trad. V. Zenatti, Paris, Éditions de l'Olivier, 2004.

2. Histoire d'une vie, p. 13.

3. Ibid., p. 15.

4. Aharon Appelfeld, Beyond Despair, Fromm International, 1993 ; traduit par M. Gribinski sous le titre L'héritage nu, Paris, Éditions de l'Olivier, 2006, p. 31.

5. Journaliste, membre du parlement israélien et spécialiste de littérature juive. Galicie $1902-$ Jérusalem 1989.

6. Aharon Appelfeld, Essais à la première personne (Massot be-guf rishon), Jérusalem, WZO, 1979.

7. L'un de plus grands poètes israéliens, Kovno 1911 - Jérusalem 1970.

8. $1892-1953$.

9. Tel-Aviv, 1972.

10. Aharon Appelfeld, «Taches de mémoire » (Kitme zikkaron), Kivunim 11-12, décembre 1997, p. 63.

11. Shmuel Schneider, «Entretien avec Aharon Appelfeld»(en hébreu), Hadoar, octobrenovembre 2003.

12. Aharon Appelfeld, Hayyim shlemim, Jérusalem, Keter, 2007.

13. Ibid.p. 200.

14. Aharon Appelfeld, Layla we-'od layla, Jérusalem, Keter, 2001.

15. Pour une analyse détaillée, voir la thèse de Ktzia Alon, Le christianisme dans la mythopoétique d'Appelfeld, Université hébraïque de Jérusalem, novembre 2006.

16. Une nuit et encore une autre, p. 33.

17. Ibid.p. 154.

\section{RÉSUMÉS}

The article takes a look at what we know about the role of languages in the life of Aharon Appelfeld. He heard Yiddish only in his early childhood, as a language spoken by his grandparents, but in Israel, after the war, learning Yiddish appeared to him as an essential step in the appropriation of Hebrew. Yiddish is an integral part of his relationship to some of his masters, like Dov Sadan or Leib Rochman. His choice of Yiddish as the main subject of his academic studies was the first step of a very painful identity process. The main work concerning this language is a novel called Night After Night (2001). It is about a boarding house in Jerusalem in the fifties. The lodgers, who are all survivors of the genocide, set themselves the task of keeping Yiddish alive. The novel is full of human conflicts between myth and reality, between the fantasy of resurrecting the past and the demands of present life. For Appelfeld, the duty of memory is essential, but it does not stand before the duty of life. Appelfeld speaks perfect Yiddish, he even uses it in public when he has the chance, but he only writes in Hebrew. It is a personal identity 
choice. He transmits the memory of the Yiddish world in Hebrew, and thus defeats oblivion forever.

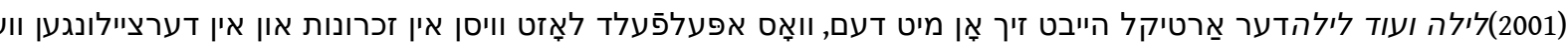

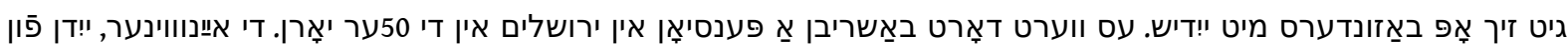

INDEX

Index chronologique : vingtième siècle

\section{מילות מפתח}

אהרן אפלפלד, החסידות, עברית, ספרות עברית, אידיש, ספרות:

Thèmes : littérature

Keywords : hasidism, Appelfeld Aharon (1932-), literature, Yiddish language Mots-clés : hassidisme, hébreu (langue), littérature hébraïque, yiddish (langue), Appelfeld Aharon (1932-) 